

\title{
Performance of the ATLAS Hadronic Tile Calorimeter
}

Tal van Daalen*, on behalf of the ATLAS Collaboration

Institut de Física d'Altes Energies (IFAE), Barcelona, Spain

E-mail: tal.van.daalen@cern.ch

The Tile Calorimeter (TileCal) of the ATLAS experiment at the LHC is the central hadronic calorimeter designed for the reconstruction of hadrons, jets, hadronically decaying tau leptons and missing transverse momentum. TileCal is a scintillator-steel sampling calorimeter and it covers the region of pseudorapidity $|\eta|<1$.7. The scintillation light produced in the scintillator tiles is transmitted by wavelength shifting fibers to photomultiplier tubes (PMTs). The analog signals from the PMTs are amplified, shaped and digitized every $25 \mathrm{~ns}$ by sampling the signal. Each step of the signal reconstruction from scintillation light to the digital pulse reconstruction is monitored and calibrated. The performance of the calorimeter has been studied in-situ employing cosmic ray muons and a large sample of proton-proton collisions acquired during the operations of the LHC. Muons of high momentum from electroweak bosons decays are employed to study the energy response of the calorimeter at the electromagnetic scale. The calorimeter response to hadronic particles is evaluated with a sample of isolated hadrons and the modelling of the response by the Monte Carlo simulation is discussed. The calorimeter timing calibration and resolutions are studied with jets. Results on the calorimeter performance on absolute energy scale, timing, noise and associated stabilities are presented. These results show that the TileCal performance is within the design requirements and has given essential contributions to the reconstruction of objects and physics results.

Sixth Annual Conference on Large Hadron Collider Physics (LHCP2018)

4-9 June 2018

Bologna, Italy

${ }^{*}$ Speaker. 


\section{Introduction}

The ATLAS detector [1] is a general-purpose detector designed to reconstruct particles originating from collisions of protons or heavy ions at the Large Hadron Collider. The Tile Calorimeter (TileCal) [2] is a hadronic calorimeter in the central region of the ATLAS detector with the main purpose of reconstructing hadrons, jets, hadronically decaying tau leptons, and missing transverse momentum. TileCal is a sampling calorimeter with steel as its main absorber and scintillating tiles as the active medium. The calorimeter is divided into a long barrel (LB) and two extended barrels (EB) with overall dimensions of $\sim 12 \mathrm{~m}$ in length and $4.25 \mathrm{~m}(2.28 \mathrm{~m})$ outer (inner) in radius, covering the region of pseudorapidity $|\eta|<1.7$. The granularity of the calorimeter corresponds to transversal segmentation of $\Delta \eta \times \Delta \phi=0.1 \times 0.1$ in the first two radial layers and $0.2 \times 0.1$ in the outermost layer. Most cells are read out by two photomultiplier tubes (PMTs), while some special cells are read out by only one PMT. TileCal uses about 10000 channels of the front-end electronics to measure the signals with energies ranging from $\sim 30 \mathrm{MeV}$ to $\sim 2 \mathrm{TeV}$.

\section{Energy reconstruction and calibration systems}

The reconstruction of the signal from the light collected by the PMTs is performed using the Optimal Filtering Technique [3], which determines the amplitude $A[\mathrm{ADC}]$, the time phase, and the pedestal of the signal from the ADC count of each channel. Next, the measured amplitude is converted into reconstructed energy $E$ using a set of calibration constants $C_{i}$, as follows:

$$
E[\mathrm{GeV}]=A[\mathrm{ADC}] \times C_{\mathrm{ADC} \rightarrow \mathrm{pC}} \times C_{\mathrm{laser}} \times C_{\mathrm{Cs}} \times C_{\mathrm{pC} \rightarrow \mathrm{GeV}}
$$

where $C_{\mathrm{ADC} \rightarrow \mathrm{pC}}, C_{\mathrm{laser}}$, and $C_{\mathrm{Cs}}$ are calibration constants derived from specific calibration systems described below, and $C_{\mathrm{pC} \rightarrow \mathrm{GeV}}$ is a factor setting the global electromagnetic scale, derived during dedicated test beams with electron energy measured [4].

The factor $C_{\mathrm{ADC} \rightarrow \mathrm{pC}}$ is determined separately for every channel using the Charge Injection System (CIS), which measures the response of the electronic channels by injecting a well determined charge into the low- and high-gain readouts. The factor is stable over time to a level of $0.02 \%$, and has a typical uncertainty of $0.7 \%$, as can be seen in Figure 1a. Front-end electronics are also monitored using the CIS such that potential instabilities or nonlinearities can be corrected.

The Laser System (LS) is used to determine the factor $C_{\text {laser }}$, and to monitor the overall stability of the gains of the PMTs in the readout chain and correct for potential gain variations. The LS injects light pulses of variable intensity into each PMT to precisely measure the response. These injections are performed during collision runs in empty bunch crossings and during dedicated laser calibration runs, which are typically taken twice a week together with CIS calibrations. The laser response evolution during the 2017 data-taking period can be seen in Figure 1b.

The Cesium calibration system is used to measure the overall response of the full readout chain and correct for potential variations with the factor $C_{\mathrm{Cs}}$. The Cesium system uses a ${ }^{137} \mathrm{Cs}$ source of $\gamma$ radiation, emitting $0.662 \mathrm{MeV}$ photons, which is moved through a series of hydraulic tubes across almost all TileCal cells to provide a signal of precisely known intensity. These measurements are subsequently used to equalize the response of each channel. 


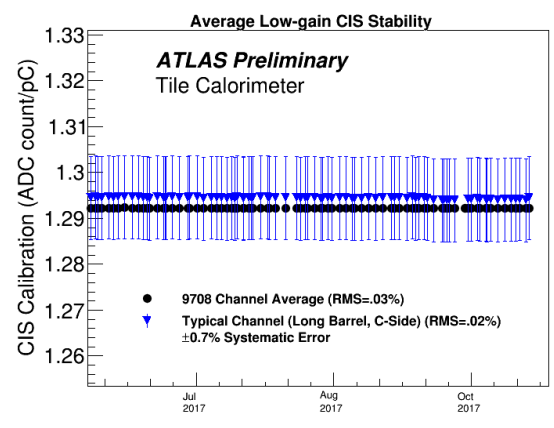

(a)

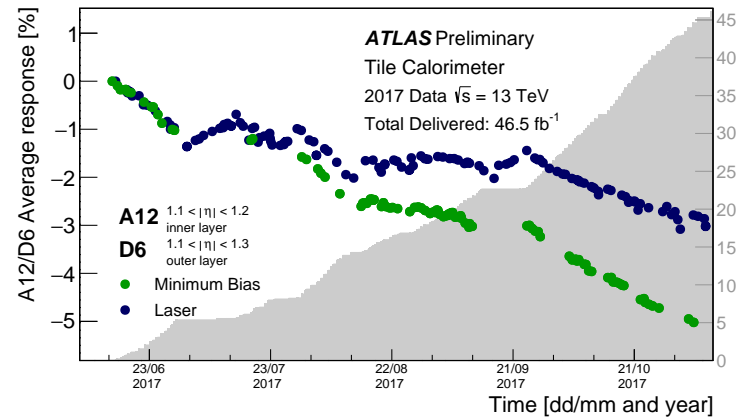

(b)

Figure 1: (a) Average of the CIS calibration constants for high-gain channels in 2017. (b) Response evolution of Minimum Bias (green) and laser (blue) for TileCal cell A12 during the 2017 datataking period, displayed as a ratio with cell D6, which is assumed to be stable over time. Figures taken from Ref. [5].

Because Cesium calibration runs cannot be taken very frequently during data-taking periods, the Minimum Bias (MB) system is designed to monitor the full readout chain during collision runs by measuring the response of each channel during minimum bias events, which are collision events with no determined selection criteria applied and therefore consist of mostly inelastic collisions. These measurements can be used as a complementary way of monitoring the response variations of each TileCal channel, but also to provide instantaneous luminosity measurements for the ATLAS detector. The MB response evolution during the 2017 data-taking period can be seen in Figure 1b, together with the laser response evolution. The difference between the laser and MB responses can be interpreted as the aging of the scintillators in the TileCal cells.

The combination of continuous monitoring and calibration systems allow TileCal to maintain a precision of the overall electromagnetic scale down to approximately $1 \%$.

\section{Performance}

Precise knowledge of the reconstruction and noise performance of TileCal is essential for the construction of topocluster information, which contributes mainly to the reconstruction of jets and missing transverse momentum in collision events. The noise in TileCal originates from static electronic noise, which is measured during special runs without collision signals, and pile-up noise, which is the dominant noise source during LHC Run 2. The reconstruction performance of Tile$\mathrm{Cal}$ is measured on several levels with either dedicated in-situ studies or more frequent monitoring procedures, as described in the following.

A dedicated study using isolated muons from $W \rightarrow \mu v$ decays was performed with $8 \mathrm{TeV}$ data to measure muon energy deposition over the path length, $\mathrm{d} E / \mathrm{d} x$, as a function of the azimuthal angle difference $\Delta \phi$ between the muon track and the center of the TileCal cell, to obtain radial response profiles for single cells in different layers of the calorimeter. In Figure 2a the normalized truncated means of $\mathrm{d} E / \mathrm{d} x$ are displayed as a function of $\Delta \phi$. These measurements allow for corrections of non-uniformities that are much smaller than the average jet size, which is typically 
larger than the size of a single TileCal cell. The observed asymmetry in the outermost radial region was caused by a slight misalignment between TileCal and the Inner Detector (ID), which has since been corrected.

Furthermore, a dedicated study using single isolated charged hadrons was performed with $1.6 \mathrm{nb}^{-1}$ of $13 \mathrm{TeV}$ proton-proton collision data collected in 2015 to cross-validate between TileCal and the ID, and to validate Monte Carlo (MC) simulations. Figure $2 b$ shows the ratio between the deposited energy $E$ of single charged hadrons, measured by TileCal, and the momentum $p$, measured by the ID, as a function of $p$, along with simulated events from PYTHIA 8.186, with the A2 tune, and the MSTW2008 LO parton distribution function set.

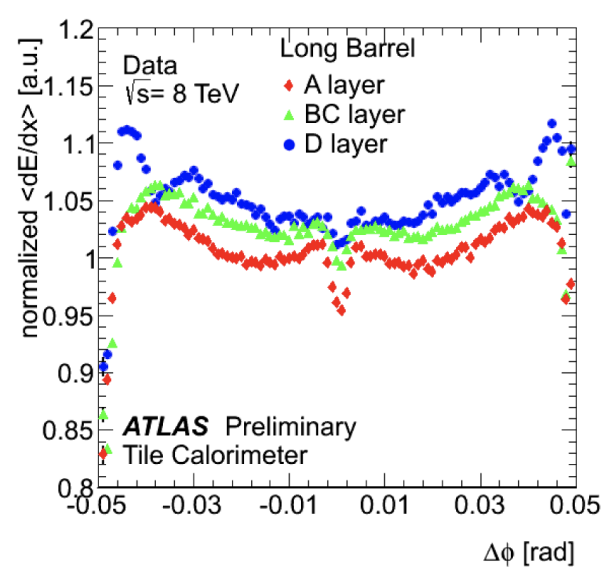

(a)

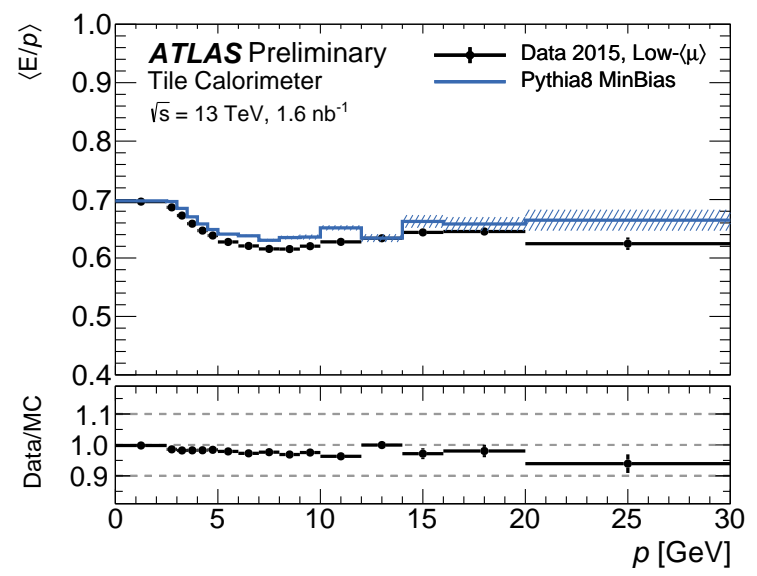

(b)

Figure 2: (a) Profiles of $\mathrm{d} E / \mathrm{d} x$ for muons from $W \rightarrow \mu v$ decays as a function of the azimuthal angle difference between the muon track and the center of the TileCal cell for different cell layers. (b) Average $E / p$ as a function of $p$ for single isolated charged hadrons for both data (black) and PYTHIA 8.186 (blue). Figures taken from Ref. [6].

The timing performance of the calorimeter is monitored and calibrated continuously using the LS in empty bunch crossings during collision runs in order to equalize the time phase of each readout channel. Figure 3 a shows the time phase in jet events for each cell as a function of the energy deposited in the cell after adjusting their global time offsets according to the time calibration system in a single reference bin (at $E \sim 21 \mathrm{GeV}$ ). The different colors correspond to different runs, and differences of up to $\sim 0.5 \mathrm{~ns}$ exist before these corrections are applied. Events with isolated jets with $p_{T}$ higher than $20 \mathrm{GeV}$ are used to minimize pile-up contamination. The time resolution is displayed in Figure $3 \mathrm{~b}$ for the same type of jet events and is smaller than $1 \mathrm{~ns}$ for jets with energies above a few $\mathrm{GeV}$. 


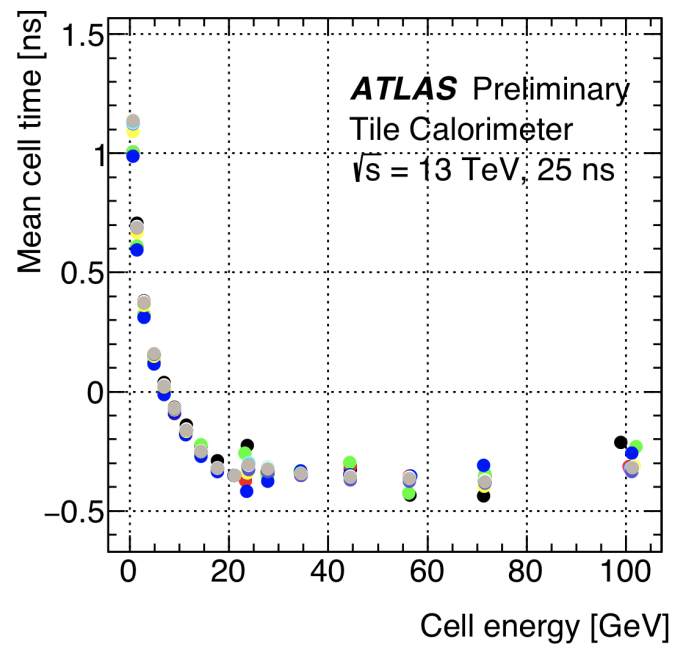

(a)

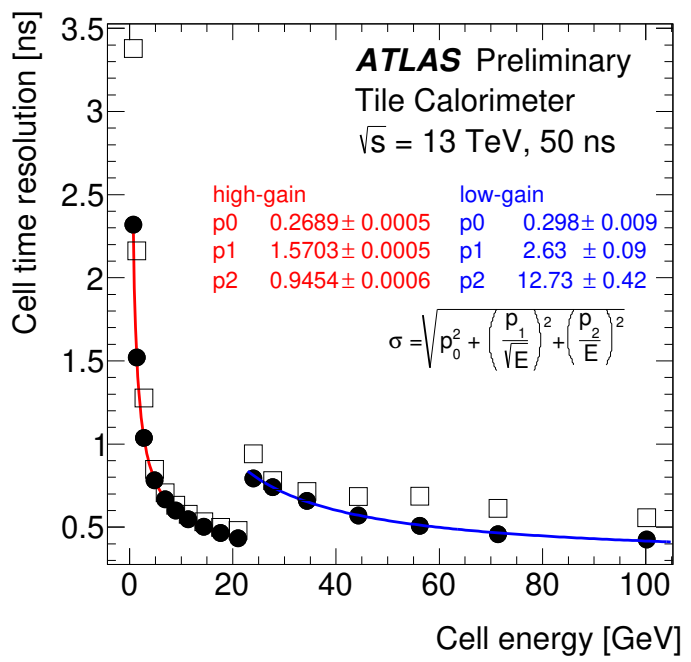

(b)

Figure 3: (a) Corrected mean cell time in jet events as a function of the energy deposited in cells. The mean cell time slowly decreases with deposited energy due to neutrons/slow hadronic component of the hadronic showers. Each colour corresponds to a different run. (b) Cell time resolution as a function of the energy deposited in LB cells, after accounting for run-to-run differences. The closed circles correspond to Gaussian $\sigma$, the open squares indicate the RMS of the underlying time distributions. Figures taken from Ref. [6].

\section{Conclusions}

The TileCal performance continues to be very stable during Run 2 of the LHC, with only $0.6 \%$ of cells masked as of April 2018. Multiple dedicated studies have been performed to measure parameters crucial to event reconstruction, to cross-validate with other detector systems, and to improve MC simulations. The several rigorous monitoring and calibration systems allow for continuous checking of the performance and stability of all components of the TileCal readout chain, and show that TileCal continues to stay within design parameters.

\section{References}

[1] ATLAS COLlaboration, Journal of Inst. 3 S08003 (2008).

[2] ATlas Collaboration, CERN LHC Exp. Comm. 96-042 (1996).

[3] W. E. Cleland and E. G. Stern, Nucl. Instrum. Meth. A 338, 46797 (1994).

[4] P. Adragna et al, Nucl. Instrum. Meth. A 606, 362 (2009).

[5] https://twiki.cern.ch/twiki/bin/view/AtlasPublic/ApprovedPlotsTile.

[6] https://twiki.cern.ch/twiki/bin/view/AtlasPublic/TileCaloPublicResults. 